\title{
The protective effect of character maturity in child aggressive antisocial behavior.
}

Nóra Kerekes ${ }^{\mathrm{a}^{*}}$, Örjan Falk ${ }^{\mathrm{b}}$, Sven Brändströmc, Henrik Anckarsäter ${ }^{\mathrm{b}}$, Maria

Råstam $^{\text {d,e }}$, Björn Hofvander ${ }^{\mathrm{d}}$

${ }^{\text {a }}$ Department of Health Sciences, University West, Trollhättan, Sweden

${ }^{\mathrm{b}}$ Centre for Ethic, Law and Mental health (CELAM), University of Gothenburg, Sweden

${ }^{\mathrm{C}}$ Center for Well-being Washington University, School of Medicine in St. Louis, USA

${ }^{\mathrm{d}}$ Department of Clinical Sciences, Lund, Child and Adolescent Psychiatry, Lund University, Sweden

${ }^{\mathrm{e}}$ Gillberg Neuropsychiatry Centre, University of Gothenburg, Sweden

*Corresponding author: Nóra Kerekes

Department of Health Sciences

University West

46186 Trollhättan

Sweden 


\begin{abstract}
Background Childhood aggressive antisocial behavior (CD) is one of the strongest predictors of mental health problems and criminal behavior in adulthood. The aim of this study was to describe personality profiles in children with CD, and determine the strength of association between defined neurodevelopmental symptoms, dimensions of character maturity and CD.
\end{abstract}

Methods A sample of 1886 children with a close to equal distribution of age (9 or 12) and gender, enriched for neurodevelopmental and psychiatric problems was selected from the nationwide Child and Adolescent Twin Study in Sweden. Their parents rated them according to the Junior Temperament and Character Inventory following a telephone interview during which information about the children's development and mental health was assessed with the Autism-Tics, AD/HD and other Comorbidities inventory.

Result Scores on the CD module significantly and positively correlated with scores on the Novelty Seeking temperament dimension and negatively with scores on character maturity (Self-Directedness and Cooperativeness). In the group of children with either neurodevelopmental or behavioral problems, the prevalence of low or very low character maturity was 50\%, while when these two problems coexisted the prevalence of low or very low character maturity increased to $70 \%$. Neurodevelopmental problems (such as: oppositional defiant disorder, symptoms of attention deficit/hyperactivity disorder and autism spectrum disorder) and low scores on character maturity emerged as independently significant predictors of CD, in a multivariable model only oppositional defiant symptoms and impulsivity significantly increased the risk for coexisting CD while a mature self-agency in a child (SelfDirectedness) remained a significant protective factor.

Conclusion These results suggest that children's willpower, the capacity to achieve personally chosen goals may be an important protective factor - even in the presence of neurodevelopmental and psychiatric problems - against progressing into persistent negative outcomes, such as aggressive antisocial behaviors. 
Keywords: character, child aggressive antisocial behavior, conduct disorder, neurodevelopment, mental health, personality, self-directedness 


\section{Introduction}

Aggressive and antisocial behavior in children and young adults is one of the strongest predictor of mental health problems and criminal behavior in adulthood (e.g. [1] Huessman et al, 2002; [2] Gelhorn et al, 2007; [3] Hofvander et al 2009, [4] Mordre et al 2011, [5] Falk et al 2014). The important question in the context of research in this area is the need to develop selection criteria to identify children and adolescents most likely to continue their anti-social behavior as adults.

\subsection{Neurodevelopment and childhood aggression}

Childhood oppositional and aggressive behaviors that override in quantity and quality the normal paths of child development are highly overlapping with each other and with other childhood mental problems ([6] Maughan et al, 2004; [7] Baker, 2013). Neurodevelopmental problems (NDPs), particularly the presence of impulsive actions/reactions and hyperactive symptoms, have been shown to increase the risk with about $80 \%$ in a child to also have symptoms of oppositional defiant disorder (ODD) and/or conduct disorder (CD) ([8] Kerekes et al 2014), and are therefore a significant predictors of childhood aggression. Deficits in maintaining attention, doubled the risk for co-existing oppositional and aggressive behavior problems in girls, but not in boys ([8] Kerekes et al, 2014). The diagnostic category AttentionDeficit/ Hyperactivity Disorder (ADHD) embraces these symptoms (impulsivity and attention problems). The high overlap between ODD/CD and ADHD is well described in different study populations (e.g.: [9] Thapar et al, 2001; [10] Kadesjö et al, 2003), and this dual diagnosis carries the highest risk for the development of persistent aggressive antisocial behavior ([3] Hofvander et al, 2009; [4] Mordre et al, 2011). Another significant neurodevelopmental problem that is associated with childhood oppositional and/or aggressive behavior is Autism Spectrum Disorder (ASD). It has been shown that two of the three symptom-clusters of ASD, i.e. deficits in social interaction and flexibility, increase the risk three to five times to also have ODD and/or CD in a child ([8] Kerekes et al, 2014). Distinct empathy- profiles have been defined in ASD children with and without CD ([11] Schwenck et al, 2012), but in each group a deficit in empathy was detected as a common underlying characteristic of social interaction problems ([12] Decety \& Moriguchi, 2007; [13] 
Woodworth \& Waschbusch, 2008). ASD itself was not found to be a risk factor for the development of aggressive antisocial behavior ([14] Palermo 2004; [15] Lundström et al, 2014). However, the prevalence of ASD in criminal populations is increased compared to the general population ([16] Kristiansson \& Sörman, 2008; [17] Billstedt et al, 2017).

\subsection{The determinants and measurement of temperament}

[18] T Moffitt (1993) was the first to introduce the expression “neuropsychological variation”, suggesting that neurological and physiological processes influence psychological characteristics, such as temperament and behavioral development, and lead to life course persistent aggressive antisocial behavior. Aggressive antisocial behavior has been associated to specific patterns of temperament and character (for review see [19] Miller and Lynam, 2001). Earlier research showed that the presence of mental problems (including aggressive antisocial behavior) is generally coupled with low character maturity ([20] Cloninger et al., 2008), while the type of problem can be characterized with specific temperament profiles ([21] Svrakic et al., 1993, [22] Garcia et al., 2013). An excellent tool to assess character and temperament profiles in order to predict the presence and eventual type of psychiatric problems is Cloninger's psychobiological model of personality ([23] Cloninger et al., 1993; [24] Cloninger et al., 1994). In this model personality comprises four temperament and three character dimensions.

Temperament is regarded as a set of biologically determined dispositions in terms of basic emotional drives and reaction patterns to stimuli, in Cloninger's model described in four dimensions which are: Novelty Seeking; Harm Avoidance; Reward Dependence and Persistence. Character traits, on the other hand, describe individual differences in higher cognitive processes reflecting a person's style of mental selfgovernment ([24, 25] Cloninger 1994a, 1994b) and were originally assumed to develop in interaction with the environment by learning. The three dimensions in the Cloninger model that capture this aspect of personality are: Self-Directedness; Cooperativeness and Self-Transcendence.

\subsection{Temperament and character in developmental psychopathology}

Several studies have investigated dimensions of temperament and character in 
young individuals with ADHD and/or ASD using the Junior Temperament and Character Inventory (J-TCI), which is based on Cloninger's model. A frequent finding is that children with ADHD, compared to typically developed children, show higher scores on Novelty Seeking, children with ASD report higher scores on Harm Avoidance, while a common profile in both ADHD and ASD show lower scores on Self- Directedness and Cooperativeness ([26] Drechsler et al., 2015; [27] Kerekes et al, 2013, [28] Josefsson et al., 2011; [29] Kim et al, 2010; [30] Purper-Ouakil et al., 2009; [31] Yoo et al., 2006). Similar findings can be seen in studies of adults with ADHD and ASD ([32] Anckarsäter et al., 2006). Lynn and colleagues (2005) [33] found that high Novelty Seeking was the best predictor of a lifetime diagnosis of ADHD compared with other temperament and character variables. The kind of personality profile that is coupled with aggressive behavior is not as widely studied as personality profiles associated with different neurodevelopmental disorder in children. In preschool populations, ODD children have been reported to share high Novelty Seeking with ADHD children, but are distinguished by their concomitant high scores in Persistence and Harm Avoidance ([34] Melegari et al., 2015). In an older sample, Drechsler and colleagues (2015) [26] found that Novelty Seeking and Persistence discriminated between children, aged 7 to 11, with ADHD and a clinical non-ADHD comparison group, but there were highly significant covariate effects of ODD/CD in terms of Novelty Seeking and Cooperativeness. Kim and colleagues (2006) [35] detected a strong link between aggressive and delinquent behaviors in adolescents (aged 13.3 \pm 0.9 years), as measured by a self-report scale, and high scores in Novelty Seeking and Harm Avoidance combined with low scores in Self-Directedness and Cooperativeness and high scores in Self-Transcendence. The same research group [29] (Kim et al., 2010) reported more extreme scores on specific personality and character dimensions in children (mean age $10.4 \pm 3.0$ years) with a combination of aggressive behavioral problems and neurodevelopmental problems (ODD and ADHD) compared to a group with "only" deviant behavior problems. In addition, the negative association between character maturity (defined by the average scores on Self-Directedness and Cooperativeness measured in the general population) and aggression has been described in violent offenders ([36] Hofvander et al., 2011, [37] Nilsson et al, 2016) and inmates generally ([38] Falk et al, 2017). However, few studies have investigated the association of childhood aggressive behavior (CD) and specific personality profiles, with a focus on the temperament constellation. One 
study found significantly increased Novelty Seeking scores in children and adolescents with CD compared to other clinical and to general population samples ([39] Schmeck and Poutska, 2001). Another study described a link between increased Novelty Seeking and neuronal correlates of emotional processing ([40] Stadler et al 2006). According to our knowledge no study has yet concentrated on the association between aggression and character in children and adolescents.

\section{Aims:}

\section{The present study sought to:}

1. measure correlations between level of child aggressive antisocial behavior (CD) and the seven dimensions of J-TCI.

2. describe and compare four groups on the prevalence of low, average and high character maturity; in children with ODD but no NDPs (i.e. ADHD or ASD), children with NDPs (i.e. (ADHD and/or ASD) but no ODD and those with ODD and comorbid NDPs (ADHD and/or ASD), and finally to a comparison group without these problems.

3. define the strength of association of independent variables: age, sex, ODD, ADHD, ASD and the dimensions of character maturity to the dependent measure of childhood aggressive antisocial behavior: CD.

\section{Methods}

\subsection{Subjects}

During the previously described ([41] Anckarsäter et al, 2011) nationwide, longitudinal Swedish twin study (the Child and Adolescent Twin Study in Sweden (CATSS)), parents of all 9- or 12-year-old twins in Sweden, since 2004, are continuously identified and contacted for a telephone interview. This interview has a strict construct of selected inventories on somatic and mental health, and includes the Autism - Tics, ADHD and other Comorbidities (A-TAC) inventory, which is described in detail below. For the present study, a follow-up questionnaire - that included among other inventories the Junior - Temperament and Character Inventory (J-TCI), described in detail below, was sent to parents of twins in whom at least one twin screened positive for ASD, ADHD, CD, ODD, tics disorder, eating disorder, developmental coordination disorder, or learning disabilities but also to parents of age- and sex-matched random control pairs, representing about 5\% of the total 
population. This questionnaire sub-study of CATSS was piloted in the total population during the first months of the CATSS (where 380 parents were contacted, of whom 196 returned complete answers) resulting in data on 392 twins (see detailed description of these pilot studies and the questionnaire sub-study in ([41] Anckarsäter et al, 2011). The overall response rate was $80 \%$ for the CATSS telephone interview, $60 \%$ for the CATSS follow-up questionnaire and 54\% for the pilot studies ([41] Anckarsäter et al, 2011). Data for the present paper were drawn from the database in January 2010. At that point data were collected from 1065 questionnaires concerning 2130 children, representing 649 screen-positive twins, 455 negative co-twins, and 692 controls, including those ( $\mathrm{n=392)}$ from the two pilot studies. In the present study, 244 children were excluded due to incomplete data, leaving 1886 children as the final study population, with a close to equal distribution of genders ( $55 \%$ boys and $45 \%$ girls) and ages (59\% were 9 years old, $41 \%$ were 12 years old).

\subsection{Instruments}

\subsubsection{Autism-Tics, AD/HD and other Comorbidities inventory}

In CATSS parents respond to the Autism - Tics, ADHD and other Comorbidities (A-TAC) inventory about their twins. This instrument includes questions formulated on the basis of DSM-IV criteria combined with clinical experiences. It covers a broad range of child psychiatric problems, all of which are answered in a life-time perspective. Responses are coded "no" (0), “yes, to some extent” (0.5), and “yes” (1.0). The instrument has been validated in cross-sectional clinical studies ([42] Hansson et al 2005; [43] Larson et al 2010) and in a longitudinal clinical follow-up study ([44] Larson et al 2014). With the help of these validated scales both continuous and categorical measures of NDPs can be measured. The continuous measures provide possibilities for analyses on the severity of psychiatric problems as well as for studies on traits of defined functional impairments, while the dichotomous measures ensure that prevalence, risk and odds calculations can be performed in this unique dataset.

The CD scale of A-TAC consists of five items with an acceptable internal reliability (Cronbach’s alpha of .61 ([41] Anckarsäter et al. 2011)). Predictive validity of the scales was high ([8] Kerekes et al, 2014) with an AUC 0.95. The present study used a cut-off of $\geq 2$ for $\mathrm{CD}$ to identify children with aggressive antisocial behavior. 
This cut-off was leads to a low sensitivity (0.55) but very high specificity (0.98) ([8] Kerekes et al, 2014).

The ODD module of A-TAC also consists of five items, and has a good internal reliability (Cronbach’s alpha of .75 ([41] Anckarsäter et al, 2011)). On this scale a cut-off of $\geq 3$ can be used to convert the ODD scores to a dichotomous category. This cut-off has previously shown a sensitivity of 0.51 and a specificity of 0.96 ([44] Larsson et al, 2014).

The ADHD scale of A-TAC contains 19 items, out of which nine correspond to the concentration/attention and 10 to the impulsiveness/activity module. The internal consistency of the complete domain is measured with a Cronbach's alpha of .92. The validated low cut-off of $\geq 6$ (with a sensitivity of .98 and a specificity of .81) ([43] Larson et al 2010) was used for screening children for ADHD in the present study.

The A-TAC modules language (five items), social interaction (six items), and flexibility (six items) can be clustered into the ASD domain, which has a good internal consistency (Cronbach's alpha of .86). The low cut-off of $\geq 4.5$ (of the maximum 17 points) that was used in the present study for screening children for ASD was previously validated and described to have very high sensitivity (0.91) and specificity (0.80) ([43] Larson et al., 2010).

\subsubsection{Junior Temperament and Character Inventory}

The Junior Temperament and Character Inventory (J-TCI), and its Swedish version, is a validated measure of children's personality profile ([45] Luby et al, 1999; [27] Kerekes et al, 2013). In the CATSS - questionnaires, the parental version of JTCI is used, which consists of 108 true/false items. Three items are so-called control questions, and 105 items assess the four temperament (Novelty Seeking, Harm Avoidance, Reward Dependence and Persistence) and the three character dimensions (Self- Directedness, Cooperativeness and Self-Transcendence). In the present study we used Self- Directedness and Cooperativeness to measure character maturity, as the construct of these two character dimensions previously has been shown to be highly relevant for children’s mental health ([27] Kerekes et al, 2013) as well as pro-social 
behavior ([46] Cloninger, 2006). This has also been described in adults ([21] Svrakic et al., 1993, [47] Richter J, Brändström S. (2009).

All participants with more than 5\% missing items on the J-TCI were excluded from the dataset. The dichotomous item construct of the J-TCI can be considered an advantage that may help to find the relevant answer, as responses in a Likert scale have a tendency to show a "regression to the mean".

\subsection{Statistical Analyses}

Non-parametric Spearman's coefficients were calculated to measure the correlation between CD scores and scores on each of the seven dimensions of J-TCI.

The prevalence of children with "normal”, "low" and "very low" character maturity (Self- Directedness and Cooperativeness) was defined with the help of Tscores. Using scores on those 691 individuals who represents the general population of 9 or 12-year-old Swedish children (366 children from the pilot studies and 325 children who were randomly selected from the general population as controls during the CATSS-questionnaires study) mean and standard deviations (SD) for each dimension of the J-TCI were calculated and then used to transform scores of each study subjects into T-scores. Normal character maturity was defined as a score between -1SD and +1SD on the sum of raw points on Self- Directedness and Cooperativeness, in other words as a T-score between 40 and 60. Low character maturity was defined as a T-score between 30 and 39, and very low character maturity was assigned to children with T-scores lower than 29.

Binary logistic regression models fitted to generalized estimated equation (GEE) - which can take the statistical dependence within twin pairs into consideration - were used to estimate the association between the dependent variable (CD) and the independent variables, ODD, activity/impulsiveness and attention (ADHD dyad) and social interaction, flexibility, and language (ASD triad), SD and CO as covariates, and age and gender as cofactors. First, univariable models were applied to study the separate effects of independent variables on CD; then multivariable models were used to investigate the effects of coexisting independent variables (ODD, activity/impulsiveness and attention problems, social interaction problems, inflexibility, language problems and character maturity (SD and CO)) on CD. All the tests were two-tailed and conducted with 5\% significance level using SPSS version 20 (IBM). 


\subsection{Ethical Consideration}

The study was designed in accordance with the Helsinki declaration and approved by the ethical review board of Karolinska Institutet (register \# 02-289 and 2010/59731/1). All analyses were performed using anonymized data files.

\section{Results}

\subsection{Correlations between child aggressive behavior and dimensions of J-TCI}

Correlation coefficients between the J-TCI dimensions and CD scores were all, except Self-Transcendence significant. While the association between CD and Harm-Avoidance, Self-Transcendence (positive), Reward-Dependence and Persistence (negative) was week, children’s aggressive antisocial behavior was moderate strongly $(/ \mathrm{r} />0.30)$ associated with Novelty Seeking (positive) and with the character dimensions Self- Directedness and Cooperativeness (negative) (Figure 1).

Figure 1 here

\subsection{Character maturity in children with neurodevelopmental and/or oppositional defiant behavioral problems}

In the control subpopulation ( $n=1281$ ) over $80 \%$ of the children had normal character maturity and less than $20 \%$ were characterized as low or very low in character maturity, suggesting significant differences in the prevalence of very low, low and normal character maturity within this group of children $(\mathrm{p}<0.001)$ (Table 1$)$.

In the subpopulation of "ODD only" ( $n=47)$ about half of the children had normal and the remains of the group had low or very low character maturity (Table 1). A similar picture was found in the subpopulation of children with "NDP only" ( $n=413$ ), where somewhat less than half of the children had normal character maturity (Table 1). In those children who had been screened both for behavioral and neurodevelopmental problems (ODD+NDP) (n=143) only one third of children had normal character maturity (Figure 2 and Table 1).

Figure 2 here 
Table 1 . The number of children with normal, low and very low character maturity (Self- Directedness and Cooperativeness) in the sub-population of children screened for NDPs and/or ODD.

\begin{tabular}{lcccccccc}
$\begin{array}{c}\text { Character } \\
\text { maturity }\end{array}$ & $\begin{array}{c}\text { Control } \\
\text { group }\end{array}$ & $\mathrm{p}$ & $\begin{array}{c}\text { ODD } \\
\text { only }\end{array}$ & $\mathrm{p}$ & $\begin{array}{c}\text { NDP } \\
\text { only }\end{array}$ & $\mathrm{p}$ & $\begin{array}{c}\text { ODD }+ \\
\text { NDP }\end{array}$ & $\mathrm{p}$ \\
\cline { 2 - 8 } $\begin{array}{l}\text { Normal } \\
\text { or high }\end{array}$ & 1077 & $<0.001$ & 25 & 0.046 & 195 & $<0.001$ & 44 & $<0.001$ \\
$\quad$ & & & & & & & \\
Low & 113 & $<0.001$ & 9 & 0.11 & 79 & $<0.001$ & 21 & $<0.001$ \\
$\begin{array}{l}\text { Very } \\
\text { low }\end{array}$ & 91 & $<0.001$ & 13 & 0.005 & 139 & $<0.001$ & 78 & $<0.001$ \\
\hline
\end{tabular}

No indication of ODD, ADHD or ASD

ODD only $=$ Children scoring at least 3 points on the ODD scale while not reaching low cut offs on ADHD neither on ASD scales of A-TAC.

NDP only = Children scoring above the lower cut-offs on ADHD and/or ASD scales but below cut-off on the ODD scale of A-TAC.

ODD+NDP = Children identified to score above the validated low cut-offs on ADHD and/or ASD as well as on ODD.

$\mathrm{p}=$ Bonferroni corrected Pearson Chi-square significance for within-group differences

\subsection{Association between NDPs, ODD, character maturity and aggressive antisocial behavior}

Age, gender and scores on ODD, facets of ADHD, facets of ASD and character maturity (Self- Directedness and Cooperativeness) were used as independent variables to analyze associations with CD diagnosis based on the previously validated cut-off. Descriptive crude measures of independent variables are shown in Table 2. The prevalence of CD was less than $4 \%(3.7 \% ; n=70)$, and it was nearly equally distributed among boys and girls (1.20:1.00) and between 9 and 12 years old children (1.45:1.00).

Table 2. Descriptive measures of the independent variables

\begin{tabular}{|c|c|c|c|c|c|c|c|c|}
\hline & & & & Crude n & asures & & & \\
\hline & & $\mathrm{n}$ & $\%$ & $\begin{array}{c}\text { \% with } \\
\text { CD }\end{array}$ & Min & Max & Mean & $\begin{array}{l}\text { Std. } \\
\text { Dev. }\end{array}$ \\
\hline age & 9 & 1117 & 59.2 & 3.9 & & & & \\
\hline & 12 & 769 & 40.8 & 3.4 & & & & \\
\hline sex & Boys & 1035 & 54.9 & 4.3 & & & & \\
\hline
\end{tabular}


ODD

ADHD

Inattention

Activity/

Impulsiveness

ASD

Language

Social

interaction

Flexibility

SD

CO
Girls

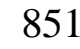

1885

1881

1883

1883

1881

1884

1881

1885

1881
45.1

3.1

$\begin{array}{cccc}0.0 & 5.0 & 0.91 & 1.21 \\ 0.0 & 19.0 & 3.9 & 4.6 \\ 0.0 & 9.0 & 2.03 & 2.55 \\ 0.0 & 10.0 & 1.85 & 2.47\end{array}$

$\begin{array}{llll}0.0 & 16.0 & 1.7 & 2.6\end{array}$

$\begin{array}{llll}0.0 & 6.0 & 0.54 & 0.96\end{array}$

$\begin{array}{llll}0.0 & 6.0 & 0.64 & 1.04\end{array}$

$\begin{array}{llll}0.0 & 5.0 & 0.55 & 0.98\end{array}$

$\begin{array}{llll}0.0 & 20 & 15.02 & 3.94\end{array}$

$0.0 \quad 20 \quad 15.61 \quad 3.59$

ODD = Oppositional Defiant Disorder; ADHD = Attention Deficit Hyperactivity Disorder; ASD = Autism Spectrum Disorder; SD = Self-Directedness; CO = Cooperativeness

While younger age and male gender somewhat increased the probability of the presence of a CD diagnosis, no significant association was found (Table 3). Both facets of ADHD (inattention and activity/impulsiveness) and all three facets of ASD (flexibility-, social interaction- and language problems) significantly increased the risk (odds) for CD, while higher scores on the character maturity dimensions of J-TCI (Self- Directedness and Cooperativeness) significantly decreased the probability that a child had co-existing CD (Table 3). In the univariate model each predictor was significantly associated with $\mathrm{CD}$, the strongest association was found for ODD $(\mathrm{OR}=3.01)$, social interaction problems $(\mathrm{OR}=2.30)$, and flexibility problems $(\mathrm{OR}=1.96)$, while Self- Directedness and Cooperativeness were the only variables that showed a negative association with $\mathrm{CD}(\mathrm{OR}=0.93$ and $\mathrm{OR}=0.94$, respectively). In the multivariate model only significant variables were included (no age and gender) and their main effect was calculated. ODD, impulsivity, inattention and social interaction problems kept their positive association with aggressive behavior, while language problems, inflexibility and character maturity dimensions (Self- Directedness and Cooperativeness) protected children from a concomitant CD diagnosis. However, only ODD, impulsivity and Self- Directedness reached a statistically significant association with CD in the multivariable model (Table 3).

Table 3. General estimated equation model $(n=1805)$ 


\begin{tabular}{lcccccc} 
& \multicolumn{3}{c}{ Univariate } & \multicolumn{3}{c}{ Multivariate } \\
\cline { 2 - 7 } & $\mathrm{p}$ & OR & $95 \%$ CI & $\mathrm{p}$ & OR & $95 \%$ CI \\
\cline { 2 - 7 } Age $^{\mathrm{a}}$ & 0.53 & 1.17 & $0.72-1.92$ & & & \\
Sex $^{\mathrm{b}}$ & 0.17 & 1.41 & $0.86-2.31$ & & & \\
ODD & $<0.001$ & 3.01 & $2.53-3.57$ & $<0.001$ & 2.09 & $1.62-2.69$ \\
Inattention & $<0.001$ & 1.49 & $1.38-1.61$ & 0.34 & 1.08 & $0.93-1.26$ \\
Impulsivity & $<0.001$ & 1.51 & $1.41-1.64$ & 0.027 & 1.16 & $1.02-1.32$ \\
Language problems & $<0.001$ & 1.73 & $1.50-1.99$ & 0.10 & 0.81 & $0.64-1.04$ \\
Social interaction & $<0.001$ & 2.30 & $2.01-2.63$ & 0.22 & 1.23 & $0.89-1.70$ \\
Inflexibility & $<0.001$ & 1.96 & $1.68-2.23$ & 0.53 & 0.90 & $0.65-1.25$ \\
Self-Directedness & $<0.001$ & 0.93 & $0.92-0.95$ & 0.035 & 0.98 & $0.96-0.99$ \\
Cooperativeness & $<0.001$ & 0.94 & $0.93-0.95$ & 0.48 & 0.99 & $0.98-1.01$ \\
\hline ODD = Oppositional Defiant Disorder & & & & \\
a = 9 years of age compared to 12; ${ }^{\text {b }}=$ boys compared to girls & & &
\end{tabular}

\section{Discussion}

In the present study, we examined the association between child aggressive antisocial behavior and the temperament and character dimensions according to Cloninger's personality model and the moderating effects of NDPs and ODD in a large sample of children that were screen-positive for different neurodevelopmental and psychiatric conditions and healthy controls.

In terms of temperament, the present study confirms the strong associations found in earlier studies between Novelty Seeking and CD ([39] Schmeck \& Poustka, 2001; [26] Drechsler et al., 2015; [35] Kim et al., 2006; [34] Melegari et al., 2015). The positive association between Harm Avoidance, which measures introvert behavior, shyness, fearfulness, excessive worrying, and highly associated to neuroticism, and CD, was not as strong as in the study by Kim and others (2006) [35]. However, that study used an older group, which could explain the discrepancy, since studies of adults with antisocial behavior have found conflicting results in terms of the level of Harm Avoidance, (e.g. [48] Snowden \& Gray, 2010; [49] Basoglu et al., 2011). We also found a negative relationship between CD, Reward Dependence and Persistence, which is in line with previous findings ([29] Kim et al., 2010; [50] Lennox \& Dolan, 2014). The combination of high Novelty Seeking and low Reward Dependence and Persistence is a well-described temperament profile that seem to carry the highest risk for the co-existence or later development of disruptive behavior disorders ([39] Schmeck \& Poustka, 2001). However, they also found low HA to be 
significantly correlated with aggression and delinquency, which confirms the need of further studies to disentangle the relationship between these two domains.

As anticipated, the measures of Self-Directedness and Cooperativeness showed the strongest negative relations to aggressive behavior. Low SelfDirectedness and Cooperativeness reflect a lacking sense of responsibility, low selfcontrol, and deficits in social skills such as tolerance to others, empathy, and the ability to be helpful and compassionate and these finding illustrate the clear association between disruptive behavior in young persons and the inability to influence one's own behavior and interpersonal skills ([22] Garcia et al., 2013)

About half of the children with oppositional behavior problems showed a low degree of character maturity. The children with NDPs (primarily ADHD and/or ASD,) showed a high degree of similarity to children with ODD in terms of character. In line with previous findings ([29] Kim et al., 2010), the comorbid condition of ODD and NDP resulted in an even more immature personality compared to children with a single problem constellation. In the group with a combination of disruptive behavior and neurodevelopmental problems, these character deficiencies were very pronounced and three out of four children in this group showed an immature profile.

Thus, the results of the present study confirm previous findings ([51] Saylor \& Amann, 2016) that a subgroup of individuals, with a combination of developmental deficiencies in the areas of impulse control, social interaction, oppositional and defiant behavior, constitute a distinct high-risk population for aggressive behavior. Moreover, we could show that normal character maturity in children with coexisting ODD, ADHD and/or ASD can serve as a protection from aggressive antisocial behavior. While our study suggests that children's level of self-directedness, as a measure of the self as an autonomous, mature individual, is the most important protective factor against aggressive and antisocial behavior, a recent study in an offender population found that in adults, the level of cooperativeness, such as social acceptance and the capacity of mentalization, may prevent individuals from criminal recidivism and persistent aggressive antisocial behavior ([38] Falk et al., 2017).

Together, these results suggest that a mature character (the willpower, the capacity to achieve personally chosen goals and the ability to adapt, integrate and 
work together with others) in children and adults can be an important protective factor - even in the presence of mental ill-health - to progress into persistent negative outcomes, such as aggressive antisocial behaviors.

\subsection{Strength and Limitations}

The present study analyzed data from a nation-wide population of 9 or 12 years old children. To work with data from the general population is important, since the results are highly generalizable. However, the low prevalence of the targeted problems in the general population resulted in small numbers of children in the specific subgroups of this study (e.g.: ODD only $n=47, C D n=70$ ).

Another limitation of our study is that all information about the children was collected from the parents. Previous studies have pointed out that when comparing information about children's/ adolescence's mental health and emotional state, the correlation between the children's own reports and their parents' scoring were weak or moderate ([52] Verhulst and Ende, 1992; [53] van Roy et al, 2010). However, the A-TAC parental interview instrument has previously shown excellent diagnostic utility to screen for children with ADHD, ASD ([42] Hanson et al, 2005, [43] Larson et al, 2010) and for those with CD, ODD ([8] Kerekes et al 2014).

Another limitation of the present study is that it relies on a study population of twins. It has previously been suggested that twin children might have a lower rate of behavior problems ([54] Moilanen et al. 1999) and they might have a lower risk to develop substance abuse or criminality than age-matched singletons ([55] Hjern et al, 2012). This limitation together with the fact that we have used cutoffs with low sensitivity, but very high specificity to select for children with CD and ODD, could carry a risk of an underestimation of the prevalence of behavior problems in our study population. In the prediction model (logistic regression), we have used the general estimated equation to balance a possible bias between independent and dependent variables of twin-pairs.

\section{Conclusion and Clinical Implications}

The confirmation of the link between character immaturity and aggression, independent of neurodevelopmental problems, in a young population is very important. While adjunctive therapy, specifically targeting aggression, often is recommended if aggressive antisocial behaviors are not altered during a primary 
ADHD therapy, there is today no empirical evidence supporting the use of any agent specific for anti-aggressive treatment ([51] Saylor and Amann, 2016). The results of our study therefore carry high relevance for the development of aggression specific treatment strategies, and suggest interventions aiming to facilitate character maturation. 


\section{References}

[1] Huesmann LR, Eron LD, Dubow EF. Childhood predictors of adult criminality: are all risk factors reflected in childhood aggressiveness? Criminal Behaviour and Mental Health. 2002;12:185-208.10.1002/cbm.496

[2] Gelhorn HL, Sakai JT, Price RK, Crowley TJ. DSM-IV conduct disorder criteria as predictors of antisocial personality disorder. Comprehesive Psychiatry. 2007;48:529-38.10.1016/j.comppsych.2007.04.009

[3] Hofvander B, Ossowski D, Lundstrom S, Anckarsater H. Continuity of aggressive antisocial behavior from childhood to adulthood: The question of phenotype definition. International Jounal Law Psychiatry. 2009;32:22434.10.1016/j.ijlp.2009.04.004

[4] Mordre M, Groholt B, Kjelsberg E, Sandstad B, Myhre AM. The impact of ADHD and conduct disorder in childhood on adult delinquency: a 30 years follow-up study using official crime records. BMC Psychiatry. 2011;11:57.10.1186/1471-244X$11-57$

[5] Falk O, Wallinius M, Lundstrom S, Frisell T, Anckarsater H, Kerekes N. The 1\% of the population accountable for $63 \%$ of all violent crime convictions. Social Psychiatry Psychiatric Epidemiology. 2014;49:559-71.10.1007/s00127-013-0783-y

[6] Maughan B, Rowe R, Messer J, Goodman R, Meltzer H. Conduct disorder and oppositional defiant disorder in a national sample: developmental epidemiology. Journal of Child Psychology and Psychiatry. 2004;45:609-21.10.1111/j.14697610.2004.00250.x

[7] Baker K. Conduct disorders in children and adolescents. Paediatrics and Child Health. 2013;23:24-9.10.1016/j.paed.2012.09.007

[8] Kerekes N, Lundstrom S, Chang Z, Tajnia A, Jern P, Lichtenstein P, et al. Oppositional defiant- and conduct disorder-like problems: neurodevelopmental predictors and genetic background in boys and girls, in a nationwide twin study. PeerJ. 2014;2:e359.10.7717/peerj.359

[9] Thapar A. Examining the comorbidity of ADHD-related behaviours and conduct problems using a twin study design. The British Journal of Psychiatry. 2001;179:2249.10.1192/bjp.179.3.224

[10] Kadesjö C, Hägglöf B, Kadesjö B, Gillberg C. Attention-deficit- hyperactivity disorder with and without oppositional defiant disorder in 3- to 7-year-old children. Developmental Medicine \& Child Neurology. 2003;45:6939.10.1017/s0012162203001282

[11] Schwenck C, Mergenthaler J, Keller K, Zech J, Salehi S, Taurines R, et al. Empathy in children with autism and conduct disorder: group-specific profiles and developmental aspects. Journal of Child Psychology and Psychiatry. 2012;53:6519.10.1111/j.1469-7610.2011.02499.x 
[12] Decety J, Moriguchi Y. The empathic brain and its dysfunction in psychiatric populations: implications for intervention across different clinical conditions. Biopsychosocial Medicine. 2007;1:22.10.1186/1751-0759-1-22

[13] Woodworth M, Waschbusch D. Emotional processing in children with conduct problems and callous/unemotional traits. Child Care Health Development. 2008;34:234-44.10.1111/j.1365-2214.2007.00792.x

[14] Palermo MT. Pervasive developmental disorders, psychiatric comorbidities, and the law. International Journal of Offender Therapy and Comperative Criminology. 2004;48:40-8.10.1177/0306624X03257713

[15] Lundstrom S, Forsman M, Larsson H, Kerekes N, Serlachius E, Langstrom N, et al. Childhood neurodevelopmental disorders and violent criminality: a sibling control study. Journal of Autism and Developmental Disorders. 2014;44:270716.10.1007/s10803-013-1873-0

[16] Kristiansson M, Sörman K. Autism spectrum disorders - Legal and forensic psychiatric aspects and reflections. Clinical Neuropsychiatry. 2008;5:55-61

[17] Billstedt E, Anckarsäter H, Wallinius M, Hofvander B. Neurodevelopmental disorders in young violent offenders: Overlap and background characteristics. Psychiatry Research. 2017;252:234-41.10.1016/j.psychres.2017.03.004

[18] Moffitt TE. Adolescence-limited and life-course-persistent antisocial behavior: a developmental taxonomy. Psychologycal Review. 1993;100:674-701

[19] Miller JD, Lynam D. Structural Models of Personality and Their Relation to Antisocial Behavior: A Meta-Analytic Review. Criminology. 2001;39:76598.10.1111/j.1745-9125.2001.tb00940.x

[20] Cloninger CR, Svrakic DM. Personality disorders. 2008.

[21] Svrakic DM, Whitehead C, Przybeck TR, Cloninger CR. Differential-Diagnosis of Personality-Disorders by the 7-Factor Model of Temperament and Character. Archives of General Psychiatry. 1993;50:991-9

[22] Garcia D, Anckarsater H, Lundstrom S. Self-directedness and cooperativeness, psychosocial dysfunction and suffering in ESSENCE. Scientific World Journal. 2013;2013:416981.10.1155/2013/416981

[23] Cloninger CR, Svrakic DM, Przybeck TR. A psychobiological model of temperament and character. Archives of General Psychiatry. 1993;50:975-90

[24] Cloninger CR, Przybeck TR, Svrakic DM, Wetzel RD. The temperament and character inventory (TCI): A guide to its development and use. St. Louis: Washington University Center for Psychobiology of Personality1994

[25] Cloninger CR. Temperament and personality. Current Opinion in Neurobiology. 1994;4:266-73.10.1016/0959-4388(94)90083-3 
[26] Drechsler R, Zulauf Logoz M, Walitza S, Steinhausen HC. The Relations Between Temperament, Character, and Executive Functions in Children With ADHD and Clinical Controls. Journal of Attention Disorders.

2015.10.1177/1087054715583356

[27] Kerekes N, Brandstrom S, Lundstrom S, Rastam M, Nilsson T, Anckarsater H. ADHD, autism spectrum disorder, temperament, and character: phenotypical associations and etiology in a Swedish childhood twin study. Comprehensive Psychiatry. 2013;54:1140-7.10.1016/j.comppsych.2013.05.009

[28] Josefsson K, Cloninger CR, Hintsanen M, Jokela M, Pulkki-Raback L, Keltikangas-Jarvinen L. Associations of personality profiles with various aspects of well-being: a population-based study. Journal of Affect Disorders. 2011;133:26573.10.1016/j.jad.2011.03.023

[29] Kim HW, Cho SC, Kim BN, Kim JW, Shin MS, Yeo JY. Does oppositional defiant disorder have temperament and psychopathological profiles independent of attention deficit/hyperactivity disorder? Comprehensive Psychiatry. 2010;51:4128.10.1016/j.comppsych.2009.09.002

[30] Purper-Ouakil D, Cortese S, Wohl M, Aubron V, Orejarena S, Michel G, et al. Temperament and character dimensions associated with clinical characteristics and treatment outcome in attention-deficit/hyperactivity disorder boys. Comprehensive Psychiatry. 2010;51:286-92.10.1016/j.comppsych.2009.08.004

[31] Yoo HJ, Kim M, Ha JH, Chung A, Sim ME, Kim SJ, et al. Biogenetic temperament and character and attention deficit hyperactivity disorder in Korean children. Psychopathology. 2006;39:25-31.10.1159/000089660

[32] Anckarsater H, Stahlberg O, Larson T, Hakansson C, Jutblad SB, Niklasson L, et al. The impact of ADHD and autism spectrum disorders on temperament, character, and personality development. American Journal of Psychiatry. 2006;163:123944.10.1176/appi.ajp.163.7.1239

[33] Lynn DE, Lubke G, Yang M, McCracken JT, McGough JJ, Ishii J, et al. Temperament and character profiles and the dopamine D4 receptor gene in ADHD. American Journal of Psychiatry. 2005;162:906-13.10.1176/appi.ajp.162.5.906

[34] Melegari MG, Nanni V, Lucidi F, Russo PM, Donfrancesco R, Cloninger CR. Temperamental and character profiles of preschool children with ODD, ADHD, and anxiety disorders. Comprehensive Psychiatry. 2015;58:94101.10.1016/j.comppsych.2015.01.001

[35] Kim SJ, Lee SJ, Yune SK, Sung YH, Bae SC, Chung A, et al. The relationship between the biogenetic temperament and character and psychopathology in adolescents. Psychopathology. 2006;39:80-6.10.1159/000090597

[36] Hofvander B, Stahlberg O, Nyden A, Wentz E, degl'Innocenti A, Billstedt E, et al. Life History of Aggression scores are predicted by childhood hyperactivity, conduct disorder, adult substance abuse, and low cooperativeness in adult psychiatric patients. Psychiatry Research. 2011;185:280-5.10.1016/j.psychres.2010.05.008 
[37] Nilsson T, Falk O, Billstedt E, Kerekes N, Anckarsater H, Wallinius M, et al. Aggressive Antisocial Behaviors Are Related to Character Maturity in Young Swedish Violent Offenders Independent of ADHD. Frontiers in Psychiatry. 2016;7:185.10.3389/fpsyt.2016.00185

[38] Falk O, Sfendla A, Brandstrom S, Anckarsater H, Nilsson T, Kerekes N. Personality and trait aggression profiles of male and female prison inmates. Psychiatry Research. 2017;250:302-9.10.1016/j.psychres.2016.12.018

[39] Schmeck K, Poustka F. Temperament and disruptive behavior disorders. Psychopathology. 2001;34:159-63.49300

[40] Stadler C, Sterzer P, Schmeck K, Krebs A, Kleinschmidt A, Poustka F. Reduced anterior cingulate activation in aggressive children and adolescents during affective stimulation: association with temperament traits. Jounal of Psychiatry Research. 2007;41:410-7.10.1016/j.jpsychires.2006.01.006

[41] Anckarsater H, Lundstrom S, Kollberg L, Kerekes N, Palm C, Carlstrom E, et al. The Child and Adolescent Twin Study in Sweden (CATSS). Twin Research and Human Genetic. 2011;14:495-508

[42] Hansson SL, Svanstrom Rojvall A, Rastam M, Gillberg C, Gillberg C, Anckarsater H. Psychiatric telephone interview with parents for screening of childhood autism - tics, attention-deficit hyperactivity disorder and other comorbidities (A-TAC): preliminary reliability and validity. British Journal of Psychiatry. 2005;187:262-7.10.1192/bjp.187.3.262

[43] Larson T, Anckarsater H, Gillberg C, Stahlberg O, Carlstrom E, Kadesjo B, et al. The autism--tics, AD/HD and other comorbidities inventory (A-TAC): further validation of a telephone interview for epidemiological research. BMC Psychiatry. 2010;10:1.10.1186/1471-244X-10-1

[44] Larson T, Kerekes N, Selinus EN, Lichtenstein P, Gumpert CH, Anckarsater H, et al. Reliability of Autism-Tics, AD/HD, and other Comorbidities (A-TAC) inventory in a test-retest design. Psychological Reports. 2014;114:93103.10.2466/03.15.PR0.114k10w1

[45] Luby JL, Svrakic DM, McCallum K, Przybeck TR, Cloninger CR. The Junior Temperament and Character Inventory: preliminary validation of a child self-report measure. Psychological Repots. 1999;84:1127-38.10.2466/pr0.1999.84.3c.1127

[46] Cloninger CR. The science of well-being: an integrated approach to mental health and its disorders. World Psychiatry. 2006;5:71-6

[47] Richter J, Brandstrom S. Personality disorder diagnosis by means of the Temperament and Character Inventory. Comprehensive Psychiatry. 2009;50:34752.10.1016/j.comppsych.2008.09.002

[48] Snowden RJ, Gray NS. Temperament and character as a function of psychopathy: relationships between the Psychopathy Checklist - Revised and the Temperament and Character Inventory in a sample of personality disordered serious 
or repeat offenders. Journal of Forensic Psychiatry \& Psychology. 2010;21:81533.10.1080/14789949.2010.506617

[49] Basoglu C, Oner O, Ates A, Algul A, Bez Y, Ebrinc S, et al. Temperament traits and psychopathy in a group of patients with antisocial personality disorder.

Comprehensive Psychiatry. 2011;52:607-12.10.1016/j.comppsych.2011.01.003

[50] Lennox C, Dolan M. Temperament and character and psychopathy in male conduct disordered offenders. Psychiatry Research. 2014;215:70610.10.1016/j.psychres.2014.01.019

[51] Saylor KE, Amann BH. Impulsive Aggression as a Comorbidity of AttentionDeficit/Hyperactivity Disorder in Children and Adolescents. Journal of Child and Adolescent Psychopharmacology. 2016;26:19-25.10.1089/cap.2015.0126

[52] Verhulst FC, Ende J. Agreement Between Parents' Reports and Adolescents' Self-reports of Problem Behavior. Journal of Child Psychology and Psychiatry. 1992;33:1011-23.10.1111/j.1469-7610.1992.tb00922.x

[53] Van Roy B, Groholt B, Heyerdahl S, Clench-Aas J. Understanding discrepancies in parent-child reporting of emotional and behavioural problems: Effects of relational and socio-demographic factors. BMC Psychiatry. 2010;10:56.10.1186/1471-244X-1056

[54] Moilanen I, Linna SL, Ebeling H, Kumpulainen K, Tamminen T, Piha J, et al. Are twins' behavioural/emotional problems different from singletons'? Europian Child and Adolescent Psychiatry. 1999;8 Suppl 4:62-7.10.1007/PL00010702

[55] Hjern A, Ekeus C, Rasmussen F, Lindblad F. Educational achievement and vocational career in twins - a Swedish national cohort study. Acta Paediatrica. 2012;101:591-6.10.1111/j.1651-2227.2012.02636.x 
Figure 1. Correlation between temperament and character dimensions of personality measured by J-TCI and aggressive antisocial behavior of children measured by CD.

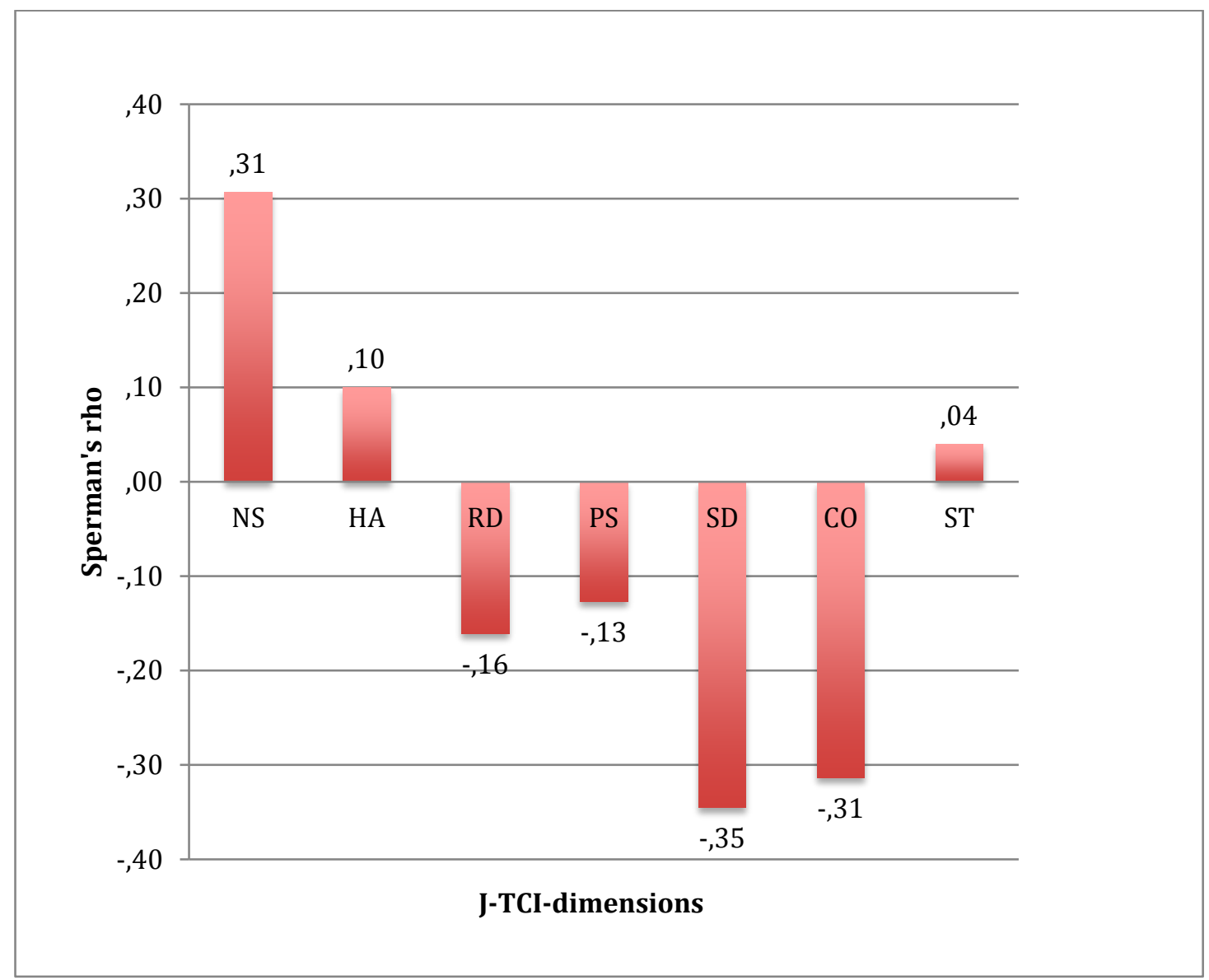

$\mathrm{NS}$ = novelty seeking, $\mathrm{HA}=$ harm avoidance, $\mathrm{RD}$ = reward dependence, $\mathrm{P}=$ persistence, $\mathrm{SD}=$ self-directedness, $\mathrm{CO}=$ cooperativeness, $\mathrm{ST}$ = self-transcendence 
Figure 2. The prevalence of normal, low and very low character maturity $(\mathrm{SD}+\mathrm{CO})$ in the sub-population of children screened for NDPs and/or ODD.

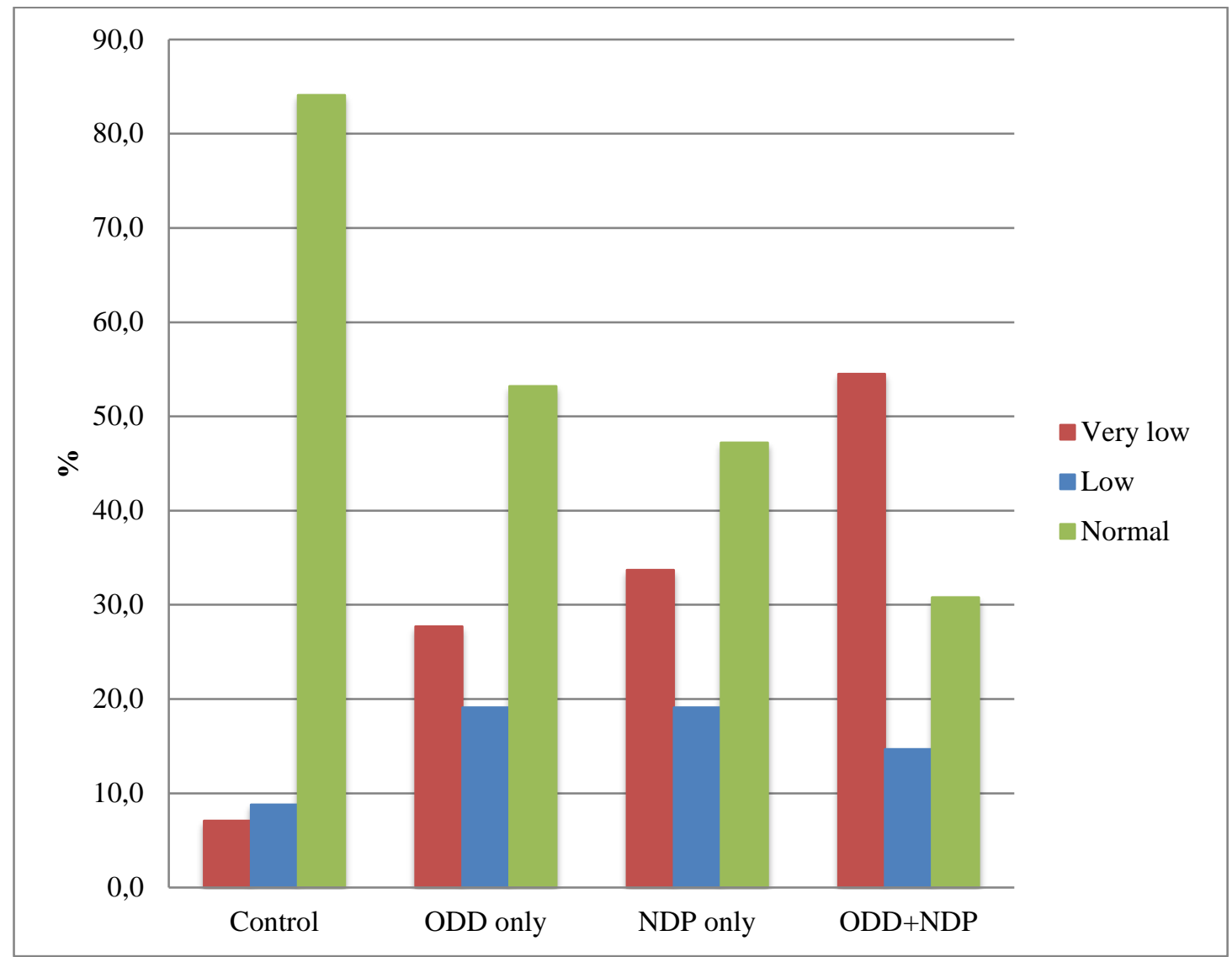

Control $=$ No indication of ODD, ADHD or ASD.

ODD only = Children scoring at least 3 points on the ODD scale while not reaching low cut offs on ADHD neither on ASD scales of A-TAC.

NDP only = Children scoring above the lower cut-offs on ADHD and/or ASD scales but below cut-off on the ODD scale of A-TAC.

ODD+NDP = Children identified to score above the validated low cut-offs on ADHD and/or ASD as well as on ODD. 